\title{
Ordered structures in rotating ultracold Bose gases
}

\author{
N. Barberán, ${ }^{1}$ M. Lewenstein, ${ }^{2,3}$ K. Osterloh, ${ }^{3}$ and D. Dagnino ${ }^{1}$ \\ ${ }^{1}$ Departamento ECM, Facultat de Física, Universitat de Barcelona, E-08028 Barcelona, Spain \\ ${ }^{2}$ ICREA and ICFO-Institut de Ciències Fotóniques, Avenida del Canal Olímpico s/n, 08860 Castelldefells, Barcelona, Spain \\ ${ }^{3}$ Institute for Theoretical Physics, University of Hannover, Appelstrasse 2, 30167 Hannover, Germany
}

(Received 13 March 2006; published 27 June 2006)

\begin{abstract}
Two-dimentional systems of trapped samples of few cold bosonic atoms submitted to strong rotation around the perpendicular axis may be realized in optical lattices and microtraps. We investigate theoretically the evolution of ground state structures of such systems as the rotational frequency $\Omega$ increases. Various kinds of ordered structures are observed. In some cases, hidden interference patterns exhibit themselves only in the pair correlation function; in some other cases explicit broken-symmetry structures appear that modulate the density. For $N<10$ atoms, the standard scenario, valid for large sytems is absent, and is only gradually recovered as $N$ increases. On the one hand, the Laughlin state in the strong rotational regime contains ordered structures much more similar to a Wigner molecule than to a fermionic quantum liquid. On the other hand, in the weak rotational regime, the possibility to obtain equilibrium states, whose density reveals an array of vortices, is restricted to the vicinity of some critical values of the rotational frequency $\Omega$.
\end{abstract}

DOI: 10.1103/PhysRevA.73.063623

PACS number(s): 03.75.Lm, 73.43.-f, 03.75.Kk

\section{INTRODUCTION}

\section{A. Ordered structures in ultracold gases and their detection}

Ordered structures, and in particular hidden ordered structures, have been a subject of intensive studies in the physics of Bose-Einstein condensates (BECs) [1-4], and more generally, in the physics of ultracold atoms.

The paradigm example of such structures is realized in the interference of two BECs, observed in seminal experiments of Ref. [5]. Suppose that, despite the superselection rule, one could prepare the two condensates in coherent atomic states, characterized by fluctuating atom numbers $N_{1}, N_{2}$, but sharply defined phases $\phi_{1}, \phi_{2}$, minimizing the Heisenberg uncertainty relation for the number and phase operators. Then, the phase difference $\Delta \phi=\phi_{1}-\phi_{2}$ would determine the position of the interference fringes. Similarly, if we prepared two condensates by, say, splitting a parent condensate with fixed number of atoms $N=N_{1}+N_{2}$, we would arrive at sharp values of both $\Delta \phi$ and $N_{1}-N_{2}$. Amazingly, the interference pattern will also appear if the two BECs in the Fock states (with fixed $N_{1}$ and $N_{2}$ ) overlap. The reason is, as pointed out in Refs. [6,7], that as soon as we start detecting atoms without knowing which condensate they originate from, the measurement will introduce the necessary uncertainty of the atom numbers, narrowing the relative phase distribution. As a consequence, an interference pattern with a sharply defined $\Delta \phi$ is obtained in each realization of the measurement. We may say that the measurement process uncovers the otherwise hidden interference pattern in the two-point first order correlation function of atomic creation and annihilation field operators, $\left\langle\hat{\Psi}^{\dagger}(\mathbf{r}) \hat{\Psi}\left(\mathbf{r}^{\prime}\right)\right\rangle$. If experimentally averaged over many realizations the interference pattern vanishes, since each realization leads to a different and completely random $\Delta \phi$. Similar measurement induced structures, and the interplay between single shot and averaged results, have also been discussed in the context of dark solitons in BEC [8].

Other types of ordered structures occur in rotating BECs. In the standard scenario, as the rotational frequency in- creases, more and more vortices appear in the form of regular structures $[4,9,10]$. As their number grows, they organize themselves in a triangular Abrikosov lattice [11]. Note that in principle the ground state of the rotating system in a harmonic trap should ideally be rotationally invariant and have a fixed total angular momentum $L$, ergo it should not exhibit any structures that break rotational symmetry, as the Abrikosov lattice does. In reality though, the preparation of vortices is performed by a "laser stirring" process that breaks rotational symmetry, and introduces significant couplings between states with different total angular momenta [12]. Here, one deals with a situation in which the preparation process (which may also be regarded as a form of measurement) reveals elsewise hidden structures in the density of the condensate, i.e., in the one-point first order correlation function $\left\langle\hat{\Psi}^{\dagger}(\mathbf{r}) \hat{\Psi}(\mathbf{r})\right\rangle$.

As it is very well-known from quantum optics, measurements of first order correlation functions (first order "coherence") do not always reveal the underlying structures. In order to see them, one has to measure higher order coherences, such as second order correlation functions $\left\langle\hat{\Psi}^{\dagger}\left(\mathbf{r}_{1}\right) \hat{\Psi}^{\dagger}\left(\mathbf{r}_{2}\right) \hat{\Psi}\left(\mathbf{r}_{3}\right) \hat{\Psi}\left(\mathbf{r}_{4}\right)\right\rangle$. The paradigm example for this necessity goes back to Michelson interferometry [13] which measures first order coherences and is sensitive to atmospheric fluctuations. This deficiency of Michelson interferometry has stimulated Brown and Twiss [14] to measure the intensity-intensity correlations of the radiation coming from Sirius, which in turn allowed them to precisely determine the coherence length and the angular size of this star.

Measurements of second order correlations play an important role in the physics of ultracold gases (for earlier works on atomic beams, see [15]). The most directly measurable quantity is the density-density correlation (pair correlation function, called pc function below): $\left\langle\hat{\Psi}^{\dagger}\left(\mathbf{r}_{1}\right) \hat{\Psi}^{\dagger}\left(\mathbf{r}_{2}\right) \hat{\Psi}\left(\mathbf{r}_{2}\right) \hat{\Psi}\left(\mathbf{r}_{1}\right)\right\rangle$, which formally is the two-point second order correlation function of the atomic field operators. This function has been directly measured in a recent atom counting experiment of the Orsay group [16], for the 
first time directly demonstrating an atomic Hanbury BrownTwiss effect for thermal atoms and the second order coherence of a BEC. Earlier, a four-point second order correlation function had been measured in Hannover [17] where densitydensity correlations of interfering condensates have been monitored in order to precisely determine the phase coherence length of quasi-one-dimensional (1D) condensates, in full analogy to the Hanbury Brown and Twiss method.

Recently, yet another tool, i.e., noise interferometry, has been proposed to analyze visible and hidden structures appearing in various quantum phases of ultracold gases [18-20]. This method also allows one to determine densitydensity correlations, and has been used by several groups to study, for instance, interference of independent BECs [21], residual coherence and lattice order in Mott insulators [22], and pair correlations of fermionic atoms in a Fermi superfluid [23].

At this point it is necessary to mention that the double (spatial and temporal) Fourier transform of the pc function is known to be a dynamical structure factor [4], and is also measurable, for instance, in Bragg scattering experiments [24].

\section{B. Rapidly rotating ultracold gases}

Recently, a considerable interest has been devoted to rapidly rotating ultracold gases, which also exhibit various kinds of ordered structures, and should therefore be investigated along the lines discussed in the previous section.

Typically, one considers a quasi-two-dimensional (2D) gas in the $X Y$ plane rotating around the $Z$ axis with frequency $\Omega$, and confined in a harmonic trap of frequency $\omega_{\perp}$. As stated above, in macroscopic atomic clouds for moderate $\Omega<\omega_{\perp}$, the Abrikosov vortex lattice is formed $[9,10]$. As $\Omega$ approaches $\omega_{\perp}$, the vortex lattice melts, and the system evolves through a sequence of strongly correlated states $[25,26]$. Finally, in the regime of critical rotation, it forms a bosonic Laughlin liquid [27].

Alternatively, the various regimes of rapidly rotating gases can be described in the terminology of fractional quantum Hall effect (FQHE) theory [28]. The crucial role is played by the direct analog of the Landau level filling factor in the FQHE which can be related to the number of vortices $N_{v}$ by $\nu=N / N_{v}$ as defined in the BEC mean field description valid for large systems and moderate rotation.

The first papers on atomic systems $[25,26]$ have considered the lowest Landau level (LLL) for strong enough rotation. Recently, correlated liquids at $\nu=k / 2$ for $k=1,2,3, \ldots$ for $\nu \leqslant \nu_{c} \simeq 6-10$ have been discussed [29]. These states resemble to a great extent the states from the Rezayi-Read (RR) hierarchy [30]: $k=1$ is the Laughlin state, $k=2$ is the Moore-Read paired state [31], etc. It has been shown that the presence of but a small amount of dipole-dipole interactions unambiguously makes the $\mathrm{RR}$ state with $k=3$ the ground state at filling $\nu=3 / 2$. This state is particularly interesting, since its excitations are both fractional and non-Abelian. The validity of the LLL approximation for rotating gases is also discussed in the recent preprint [32].

Most of the literature on ultracold rapidly rotating gases aims at considering relatively large systems and even the thermodynamic limit. In numerical simulations, either periodic (torus) or spherical boundary conditions are used. Unfortunately, in the $N \rightarrow \infty$ limit the gap separating the Laughlin state from its excitations vanishes. Observation of Laughlin states not only requires one to reach the LLL, but also to control very precisely a delicate balance between $\Omega$ and $\omega_{\perp}$. Despite the progress in experimental studies of vortex lattices [33,34], and first steps towards LLL physics [35], experiments have not yet reached this regime.

The problems related to the short range nature of the Van der Waals forces can be overcome in dipolar gases, i.e., gases that interact via magnetic or electric dipole moments (for a review see [36]). Rotating dipolar bosonic gases are expected to exhibit exotic behavior in the weakly interacting regime [37], whereas fermionic dipolar gases have a finite gap for the $\nu=1 / 3$ Laughlin state [38]. The first observation of BEC of a dipolar gas of chromium atoms with large magnetic dipole has been recently reported [39], and several groups are trying to realize and control an ultracold gas of heteronuclear molecules with large electric dipole moments [40].

Another way to create highly correlated liquids could be, not to mimic effects of magnetic fields by rotation, but by appropriately designed control of tunneling phases in optical lattices [41]. In trapped gases, a similar effect may be realized by employing electromagnetically induced transparency [42].

However, the most promising way towards the $\mathrm{FQH}$ regime and related states may be achieved by use of an array of rotating optical microtraps, either in an optical lattice [43], or created by an array of rotating microlenses [44] or by manipulation of microscopic size traps created by a tightly focused laser, as in experiments of Ref. [45].

In such arrangements, it will be natural to study mesoscopic, or even microscopic systems, of few atoms. Such experiments demand careful theoretical studies of few atom systems using possibly exact methods, such as exact diagonalizations of the Hamiltonian with open boundary conditions in the presence of the harmonic trap, or even a deformed trap. Such studies have recently been initiated [46], and the possibilities of an adiabatic path to fractional quantum Hall states of a few bosonic atoms have been investigated in detail. We continue the studies of small systems of atoms in rotating traps, and expand them in the present paper.

\section{Plan of the paper}

The main focus of the present paper is to study and analyze the ground state (GS) ordered structures and interference patterns (IP) of two-dimensional Bose systems of few atoms confined in a harmonic trap in the $X Y$ plane and submitted to fast rotation around the $Z$ axis. We investigate here, on one hand, the situations where the cylindrical symmetry is explicitly broken so that the one particle density already exhibits ordered structures due to the coherent mixing of degenerated GS with different total angular momenta. Furthermore, we consider situations in which the ordered patterns are hidden in a pure single state with well-defined angular momentum, and are evident only through inspection of the 
pair correlation (pc) function calculated by means of exact diagonalization. This formalism turns out to be the appropriate method to deal with small systems, for which the assumptions made in mean field theories do not apply. Such systems are experimentally accessible, and both density and pc functions are measurable by various experimental techniques discussed in the previous section. Experimental information on the IP can be obtained in the last case.

According to our findings, the behavior of confined systems of few atoms strongly differs from the behavior of large systems. These differences are not only related to the nucleation of vortices in the regime of relatively slow rotation, but also to the nature of the Laughlin state and other highly correlated states, when the rotational frequency is close to the trap frequency.

In particular, we obtain that for $N<10$ atoms (and in some cases even for larger values of $N$ ) the standard scenario valid for large sytems (i.e., the nucleation of vortices into an Abrikosov lattice, melting of the lattice, and subsequent appearance of fractional quantum Hall type states up to the Lauhglin state) is only gradually recovered as $N$ increases. Within the regime of relatively weak rotation, the transition of the ground-state angular momentum from zero to $\mathrm{N}$ cannot be interpreted as the nucleation of the first centered vortex with circular symmetric structure that would exhibit no spacial correlation. Instead, some correlation is detected in the pc function that even survives in the case of $N=20$. Furthermore, as $\Omega$ increases, the possibility to obtain brokensymmetry ground states that would show vortex structures is restricted to the vicinity of some critical values of $\Omega$ for which degeneracy of several different eigenstates with welldefined angular momenta is possible. This situation differs from the behavior of large systems for which the amount of vortices increases monotonously while $\Omega$ increases as it has been experimentaly obtained by Chevy et al. [47]. In addition, as $\Omega$ approaches $\omega_{\perp}$, the Laughlin state reveals ordered structures in its pc function much more similar to the finite version of a Wigner crystal than to a Fermi liquid type state expected for large systems. This last result has some similarities to electronic systems, extensively analyzed previously.

This paper is organized as follows. In Sec. II we describe our system (Sec. II A) and address the questions related to the realization and analysis of ground states with hidden (Sec. II B), or explicit (Sec. II C) broken cylindrical symmetry. In Sec. III, the main results of this work are presented. Finally, in Sec. IV, we compare our findings with previous results in the literature, and draw our conclusions.

\section{ORDERED STRUCTURES IN GROUND STATES: BROKEN CYLINDRICAL SYMMETRY}

\section{A. Description of the system}

Our system consists of $N$ bosonic atoms trapped in a rotating parabolic potential. The Hamiltonian in the rotating reference frame reads [48]

$$
H=\sum_{i=1}^{N}\left[\frac{\left(\vec{p}-\frac{e}{c} \vec{A}^{*}\right)_{i}^{2}}{2 M}+\frac{1}{2} M\left(\omega_{\perp}^{2}-\Omega^{2}\right) r_{i}^{2}\right]+g \sum_{i<j} \delta\left(\vec{r}_{i}-\vec{r}_{j}\right),
$$

where $\vec{r}=(x, y), \omega_{\perp}$ is the trap frequency, $\vec{A}^{*}=(M \Omega c / e) \hat{z}$ $\times \vec{r}$ is the vector potential, $\hat{z}$ is the unitary vector along the $Z$ direction, and $\vec{B}^{*}=\vec{\nabla} \times \vec{A}^{*}=(2 M \Omega c / e) \hat{z}$ is the effective magnetic field of an equivalent system of electrons submitted to a magnetic field perpendicular to the $X Y$ plane (we use here the symmetric gauge). Thus the rotation of the trap has formally the same effect on atoms of mass $M$ as a magnetic field has on electrons; the electronic charge $-e$ and the speed of light $c$ are solely introduced for reasons of algebraic equivalence. $V=g \sum_{i<j} \delta\left(\vec{r}_{i}-\vec{r}_{j}\right)$ is the contact interaction potential, where $g$ is the interaction coefficient that approximates the potential of the Van der Waals forces between the atoms in the very dilute limit. We assume the rotational frequency to be large enough to restrict the system to the lowest Landau level (LLL) regime, and choose the appropriate Fock-Darwin single particle (sp) wave functions with no nodes in the radial direction as the basis in order to represent all operators [49],

$$
|m\rangle=\frac{1}{\lambda \sqrt{\pi m !}}\left(\frac{z}{\lambda}\right)^{m} e^{-|z|^{2} / 2 \lambda^{2}}
$$

with $\lambda=\sqrt{\hbar /\left(2 M \omega_{\perp}\right)}$, and generalized complex coordinates $z=x+i y$.

The Hamiltonian can be written in second quantized form as

$$
\hat{H}=\alpha \hat{L}+\beta \hat{N}+\hat{V},
$$

where $\alpha=\hbar\left(\omega_{\perp}-\Omega\right), \quad \beta=\hbar \omega_{\perp}, \hat{L}$ and $\hat{N}$ are the total $z$-component angular momentum and particle number operators, respectively, and

$$
\hat{V}=\frac{1}{2} \sum_{m_{1} m_{2} m_{3} m_{4}} V_{1234} a_{1}^{\dagger} a_{2}^{\dagger} a_{4} a_{3},
$$

where the matrix elements of the interaction term are given by

$$
V_{1234}=\left\langle m_{1} m_{2}|V| m_{3} m_{4}\right\rangle=\frac{g}{\lambda^{2} \pi} \frac{\delta_{m_{1}+m_{2}, m_{3}+m_{4}}}{\sqrt{m_{1} ! m_{2} ! m_{3} ! m_{4} !}} \frac{\left(m_{1}+m_{2}\right) !}{2^{m_{1}+m_{2}+1}} .
$$

Here, the operators $a_{i}^{\dagger}$ and $a_{i}$ create and annihilate a boson with single-particle ( $\mathrm{sp}$ ) angular momentum $m_{i}$, respectively. The cylindrical symmetry of the Hamiltonian allows the diagonalization to be performed in different subspaces of a well-defined total $z$-component of angular momentum $L=\sum_{i=1}^{N} m_{i}$.

Figure 1 shows the total angular momentum of the GS of a system of $N=5$ particles while $\Omega$ grows from zero to $\omega_{\perp}$, the maximum possible value before the system becomes centrifugally unstable. We observe that the GS angular momentum remains constant for a finite range of $\Omega$ until transitions 


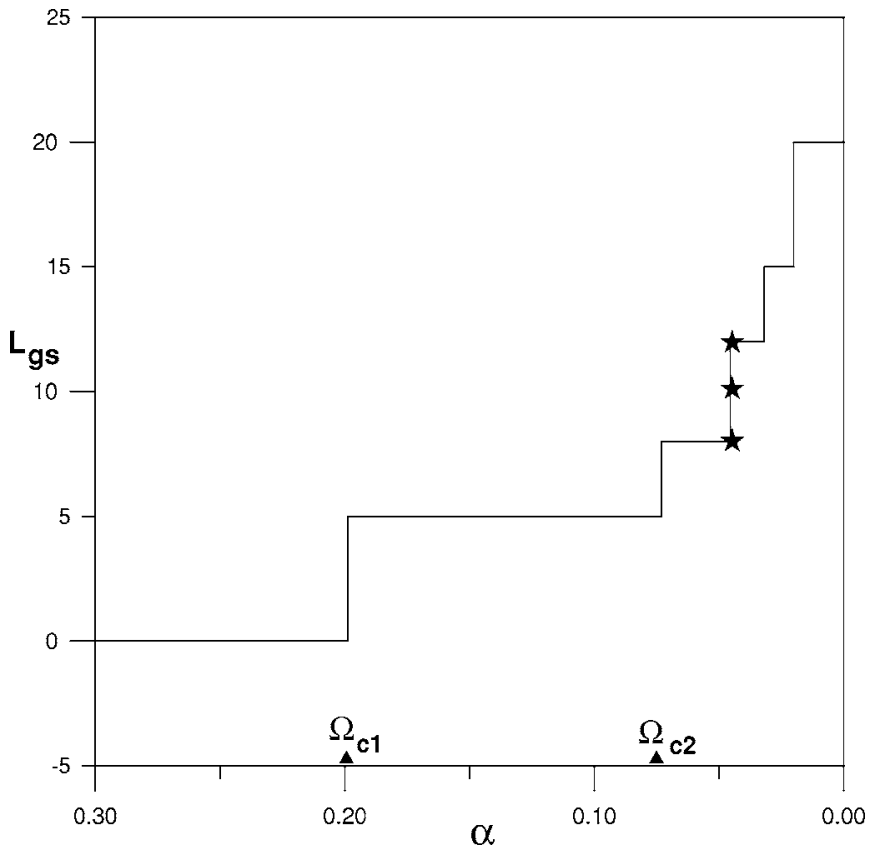

FIG. 1. Change of the GS angular momentum $L_{g s}$ for $N=5$ as the rotation frequency increases; transitions take place at critical values of the rotational frequency labeled by $\Omega_{c n}$. $\alpha$ is defined as $\alpha=\hbar\left(\omega_{\perp}-\Omega\right)$ in units of $\hbar \omega_{\perp}$; it lowers as the rotation frequency approaches $\omega_{\perp}$.

to new angular momenta take place at critical values labeled as $\Omega_{c n}$. Not all $L$-values can be associated with the GSs. However, on the steps, different $L$-states may be degenerate in energy as in the case of the states with $L=8,10$, and 12 on the third step (indicated by stars in Fig. 1). The last possible GS at $L=N(N-1)$ is the Laughlin state, for which the interaction energy is zero due to the fact that the wave function of each atom has zeros of order two at the positions of the other $N-1$ atoms; this can be easily deduced from the analytical expression of the many-body wave function given by

$$
\Psi_{\text {Laughlin }}=\mathcal{N} \prod_{i<j}\left(z_{i}-z_{j}\right)^{2} e^{-\Sigma\left|z_{i}\right|^{2} / 2 \lambda^{2}}
$$

In the following, the GS of the system in the $L$-subspace is denoted by $\Psi_{L}$. In order to study the nature of the GSs, it is useful to analyze the expectation values of some relevant operators. First of all, it is crucial to realize that the density operator defined in first quantization as

$$
\hat{\rho}(\vec{r})=\sum_{i=1}^{N} \delta\left(\vec{r}-\vec{r}_{i}\right)
$$

does not exhibit any interference pattern when calculated for a definite $\Psi_{L}$, as it can be inferred from its analytical expression in second quantized form

$$
\hat{\rho}(\vec{r})=\sum_{i j}\left\langle\phi_{i}\left(\overrightarrow{r^{\prime}}\right)\left|\delta\left(\vec{r}-\vec{r}^{\prime}\right)\right| \phi_{j}\left(\overrightarrow{r^{\prime}}\right)\right\rangle a_{i}^{\dagger} a_{j},
$$

where $\left|\phi_{i}(\vec{r})\right\rangle=\left|m_{i}\right\rangle$ as in Eq. (2). Due to angular momentum conservation, the operator $a_{i}^{\dagger} a_{j}$ selects only one $s p$ state and, as a consequence, it loses all information contained in prod- ucts of different amplitudes, thus losing the interference pattern. It solely preserves the information of individual densities, e.g.,

$$
\rho(\vec{r})=\left\langle\Psi_{L}|\hat{\rho}(\vec{r})| \Psi_{L}\right\rangle=\sum_{i}^{N}\left|\phi_{i}(\vec{r})\right|^{2} O c_{i},
$$

where $O c_{i}$ is the total occupation of the $s p$ state $\left|m_{i}\right\rangle$ in the GS. In effect, $\rho(\vec{r})$ can only represent cylindrically symmetric distributions. However, we note that this cylindrical symmetry is a direct consequence of the definition of the operator $\hat{\rho}(\vec{r})$, and is not necessarily a manifestation of the symmetric nature of the GS.

To exhibit ordered patterns and analyze the GS structures, we proceed in two different ways; one investigates the pair correlation function for states with fixed $L$ (Sec. II B), the other combines different $\Psi_{L}$ 's (Sec. II C).

\section{B. Ordered structures in pair correlation functions}

In order to analyze the internal structure of relevant states, we consider the following operator:

$$
\hat{\rho}\left(\vec{r}, \vec{r}_{0}\right)=\sum_{i<j}^{N} \delta\left(\vec{r}_{i}-\vec{r}_{0}\right) \delta\left(\vec{r}_{j}-\vec{r}\right),
$$

which yields the conditional probability to find an atom at $\vec{r}$, when another is simultaneously found at $\vec{r}_{0}$. This operator contains information that originates from the amplitudes of $s p$ wave functions, and not only from their density as it has been in the case of the single particle density operator. In second quatized formalism, its expected value with respect to $\Psi_{L}$ reads

$$
\begin{aligned}
\rho\left(\vec{r}, \vec{r}_{0}\right)= & \sum_{i j k l} \sum_{p p^{\prime}} \alpha_{p}^{*} \alpha_{p^{\prime}} \phi_{i}^{*}(\vec{r}) \phi_{j}^{*}\left(\vec{r}_{0}\right) \phi_{k}(\vec{r}) \phi_{l}\left(\vec{r}_{0}\right) \\
& \times\left\langle\Phi_{p}\left|a_{i}^{\dagger} a_{j}^{\dagger} a_{l} a_{k}\right| \Phi_{p^{\prime}}\right\rangle,
\end{aligned}
$$

where

$$
\Psi_{L}=\sum_{p=1}^{n_{d}} \alpha_{p} \Phi_{p}
$$

and $\Phi_{p}$ are the bosonic Fock $N$-body states of the basis in the $L$-subspace of dimension $n_{d}$. The condition $i+j=k+l$ must be fulfilled for reasons of angular momentum conservation. It should be stressed that $\rho\left(\vec{r}, \vec{r}_{0}\right)$ in Eq. (10) obviously differs from the single particle density matrix

$$
n^{(1)}\left(\vec{r}, \vec{r}^{\prime}\right)=\left\langle\hat{\Psi}^{+}(\vec{r}) \hat{\Psi}\left(\vec{r}^{\prime}\right)\right\rangle,
$$

which defines the off-diagonal long-range order that characterizes Bose condensation [4]. The operator $\hat{\rho}$ is a twoparticle operator, whereas $\hat{n}^{(1)}\left(\vec{r}, \vec{r}^{\prime}\right)=\hat{\Psi}^{+}\left(\vec{r}^{\prime}\right)$ is a singleparticle operator; in particular, $\rho(\vec{r})=n^{(1)}(\vec{r}, \vec{r})$, whereas $\rho(\vec{r})=\frac{1}{N-1} \int d \vec{r}_{0} \rho\left(\vec{r}, \vec{r}_{0}\right)$. As a rule of thumb, if $\hat{n}^{(1)}\left(\vec{r}, \vec{r}^{\prime}\right)$ reveals symmetry breaking, so does $\hat{\rho}$, whereas the opposite is not necessarily true.

Equation (11) can be interpreted as the sum of products of amplitudes at $\vec{r}$ weighted by a factor that depends on $\vec{r}_{0}$, and 
on the GS via the $\alpha_{p}$ coefficients. In the particular case $\vec{r}_{0}=\overrightarrow{0}$, cylindrical symmetry is recovered, since in this case $l=j=0$ is the unique nonzero contribution. This implies $i=k$ and yields

$$
\rho(\vec{r}, \overrightarrow{0})=\left|\phi_{0}(\overrightarrow{0})\right|^{2} \sum_{i}\left|\phi_{i}(\vec{r})\right|^{2} \sum_{p p^{\prime}} \alpha_{p}^{*} \alpha_{p^{\prime}}\langle\cdots\rangle,
$$

which is independent from $\theta$.

In order to understand the role of the parameter $\vec{r}_{0}$ in $\left\langle\Psi_{L}\left|\hat{\rho}\left(\vec{r}, \vec{r}_{0}\right)\right| \Psi_{L}\right\rangle$ as a function of $\vec{r}$, we consider

$$
\begin{aligned}
\phi_{n}^{*}(\vec{r}) \phi_{j}^{*}\left(\vec{r}_{0}\right) \phi_{k}(\vec{r}) \phi_{l}\left(\vec{r}_{0}\right)= & \frac{1}{\pi^{2}} \frac{r^{m_{n}}}{\sqrt{m_{n} !}} \frac{r_{0}^{m_{j}}}{\sqrt{m_{j} !}} \frac{r^{m_{k}}}{\sqrt{m_{k} !}} \frac{r_{0}^{m_{l}}}{\sqrt{m_{l} !}} e^{i\left(m_{k}-m_{n}\right) \theta} \\
& \times e^{i\left(m_{l}-m_{j}\right) \theta_{0}} e^{-r^{2}} e^{-r_{0}^{2}}
\end{aligned}
$$

in units of $\lambda$. As $l-j=n-k$ follows from angular momentum conservation, the angular dependence reads

$$
e^{i\left(m_{k}-m_{n}\right) \theta} e^{i\left(m_{l}-m_{j}\right) \theta_{0}}=e^{i\left(m_{k}-m_{n}\right)\left(\theta-\theta_{0}\right)} .
$$

Evidently, if $r_{0}$ is fixed, the change of $\theta_{0}$ is nothing but a rigid rotation of the function. In other words, any arbitrary choice of $\theta_{0}$ fixes the origin of angles, and breaks cylindrical symmetry, in the analogous way as it happens in experiments which perform a single shot measurement. Within this point of view, the experimental measurement and the choice of $\theta_{0}$ are equivalent processes (see for instance $[18,22]$ ).

The expected values of the pc function for $r_{0} \neq 0$ can reveal very different situations: from circular symmetric structures showing no spatial correlation, to ordered structures that reveal intrinsic Wigner molecules or crystals while passing through all possible intermediate states, as it is shown in Sec. III.

\section{Ordered structures in the density: Superpositions of different $L$-subspaces}

In this section, we consider ordered structures in GSs with no well-defined angular momentum in two different situations. On the one hand, we build (somewhat ad hoc) linear combinations of different $\Psi_{L}$ 's to explicitly reveal the structure present in the expectation value of the density operator. We obtain ordered IPs for combinations, whenever one of the contributing $\Psi_{L}$ 's has an ordered hidden IP contained in its pc function. On the other hand, after the introduction of an anisotropic term to the Hamiltonian which mimics the deformation introduced by the stirring laser, we perform numerical diagonalization without the restriction of angular momentum conservation, and obtain in this way GS structures with broken symmetry. These exact calculations give hints how to construct approximated GS superpositions in the previous $a d$ hoc construction.

To explain more precisely, how the first procedure works, we start from the pc function and observe what kind of ordered structures can be expected. In the case of the Laughlin state $L=20, N=5$, the pc function suggests that the atoms form a pentagon. Quite generally, the best way to visualize this structure within the first procedure is to form a superposition $A \Psi_{L}+B \Psi_{L+N}$, where $\Psi_{L}$ is the GS that contains a hid-

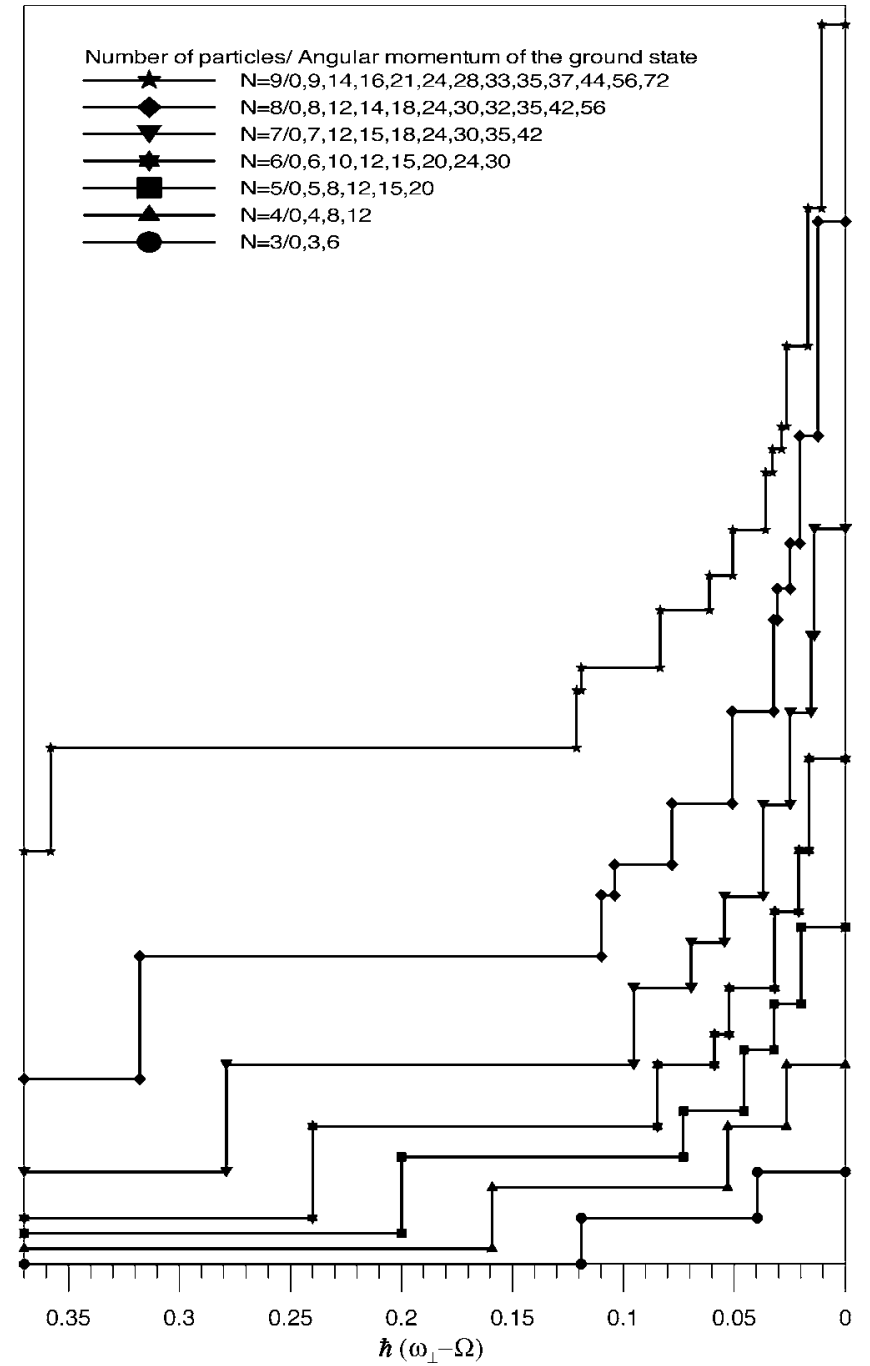

FIG. 2. The same as in Fig. 1 for $N=3$ to 9 from bottom to top. Graphs are vertically shifted for clarity. The (quasi-)degenerate states which the same step are not included.

den ordered IP. It is easy to understand this result from what follows. The terms that contribute to the broken cylindrical symmetry are those of the form $\left\langle\Psi_{L}\left|a_{i}^{\dagger} a_{j}\right| \Psi_{L+M}\right\rangle$ with $M \geqslant 1$. However, it is necessary to arrive at $M=N$ in order to obtain contributions from all the sp states contained in $\Psi_{L}$. To be more precise, none of the combinations from $L=20+21$ to $L=20+24$ reproduces the structure of $L=20$ for $N=5$. It is necessary to combine $L=20+25$ to obtain the regular pentagon implicit in $\Psi_{20}$. The best contrast is obtained for $A=B=1$, and not for a small amount of $\Psi_{L+N}$ as one would expect if only a perturbation would be necessary.

It is important to stress that the ordered hidden IP was obtained in the Laughlin state $L=20$ for $N=5$. In order to assure that this state is the GS, a very small amount of kinetic energy is necessary in such a way that the states $L+M$ for $M \geqslant 0$ are quasidegenerate. Then, the combination considered above corresponds to a "legitimate" GS.

The second procedure, followed in order to obtain ordered structures as, e.g., multiple vortex states, was suggested by experiments. In the experimental setup described by Chevy 

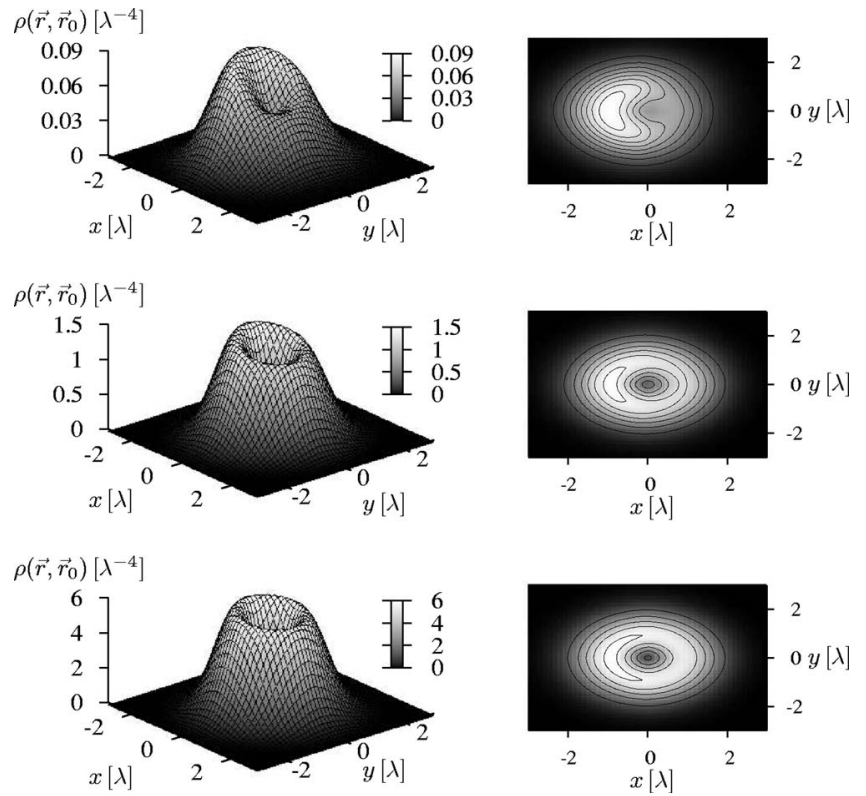

FIG. 3. Pair correlation function (3D plot and contour plot) for $N=3,10$, and 20 of the $L=N$ state. The parameter $r_{0}$ is equal to 0.8 , 0.95 , and 1.0 in units of $\lambda$, respectively.

et al. [47] and by Madison et al. [9], vortices are generated as equilibrium states of a Bose condensate rotating under the action of a stirring laser that produces anisotropy in the $X Y$ plane. Subject to this anisotropic potential, the state with vortices is a GS, and it survives during the time of flight (TOF) detection as an excited state of the restored symmetric Hamiltonian after the trap is switched off.

With this idea in mind, we introduce an additional anisotropic term in the Hamiltonian given by $\hat{V}_{p}=A \sum_{i=1}^{N}\left(x^{2}-y^{2}\right)_{i}$ or in second quantized form as [50]

$$
V_{p}=\frac{A}{2} \lambda^{2} \sum_{m}\left[\sqrt{m(m-1)} a_{m}^{+} a_{m-2}+\sqrt{(m+1)(m+2)} a_{m}^{\dagger} a_{m+2}\right] \text {. }
$$

We assume this term to be a small perturbation of the system, thus, $A \lambda^{2} /\left[2 \hbar\left(\omega_{\perp}-\Omega\right)\right]<<1$, and perform exact diagonalization to obtain the GS of $H+V_{p}$.

Amazingly, the structure of, say, two vortices can only be obtained at very specific plateau steps $\Omega_{c n}$. There, the GS is a combination of quasidegenerate $\Psi_{L^{\text {-states }}}$ which are coupled by a perturbing term slightly larger than their energy difference but much smaller than the next eigenenergy. As a consequence, the linear combination of the states above has nearly equal coefficients. Thus a direct ad hoc combination of degenerated states of the symmetric Hamiltonian of Eq. (1) are educated guesses to reveal underlying structures. This combination was previously used by Wilkin et al. in exact diagonalization calculations to obtain two vortices [25]. More precisely, the unique situation where vortices are generated in the density corresponds to the steps in the $L_{g s}$ dependence on $\Omega$, where a degeneracy of states with different $L$ takes place at $\Omega_{c n}$. At first sight, this result does not agree with the experimental results reported by Chevy et al. [47].

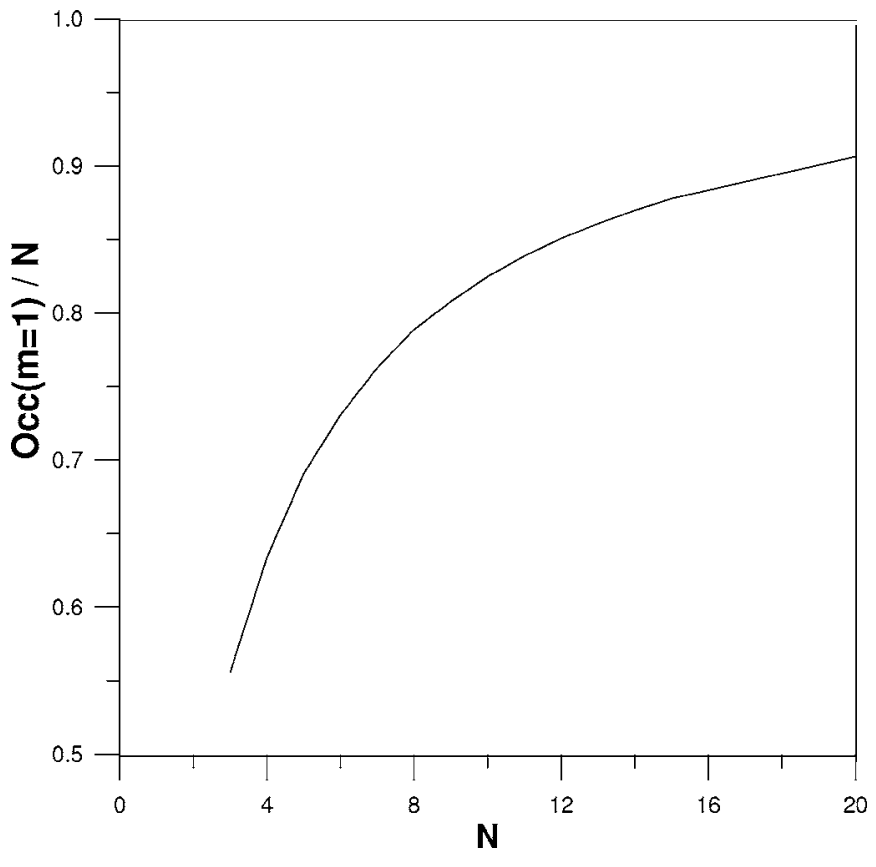

FIG. 4. Occupation of the $m=1$ single state divided by $N$ as a function of $N$.

However, it can be attributed to an essentially different behavior of systems with a large and a small number of atoms, respectively. As $N$ grows, the size of some of the plateaus shown in Fig. 1 drastically shrinks in such a way that finite ranges of $\Omega$-values with energetically degenerate states become possible; not only at critical values $\Omega_{c n}$. In Fig. 2, we show the appearance of such microplateaus obtained for $N=6,7,8$, and 9 .

Deduced from these features, our prediction is that the experimental graph, analogous to the one displayed by Chevy et al. [47] in their Fig. 2 which shows a monotonous growth of the vortex contribution to $L_{g s}$ as a function of $\Omega$, from the first vortex nucleation (at $\Omega_{c 1}$ ) to the turbulent regime, would look radically different for small $N$. We expect that it would present a curve with minima at those values of $\Omega$ where the GS is deep in a plateau, and exhibits no vortices in the density. Vortices will solely be visible on microplateaus surrounding $\Omega_{c n}$. For fixed but larger $N$, the microplateaus contain more and more states as $\Omega$ increases, and thus a larger number of vortices is nucleated. Ultimately, in the large $N$ limit, the number of vortices becomes proportional to the rotational frequency.

\section{NUMERICAL RESULTS}

In what follows, we display the results obtained from exact diagonalization for $g \lambda^{-2}=1$ in units of $\hbar \omega_{\perp}$. The values $\omega_{\perp}=\Omega=0$ were considered for density and correlation functions of single states, or combinations of states with welldefined angular momentum, as the diagonalization of $H$ depends only on the interaction energy. In contrast, specific values of $\alpha$ and $\beta$ [see Eq. (3)] are considered when the diagonalization of $H+V_{p}$ is performed. Correlation functions are always displayed in pairs, a 3D and a contour plot, unless 

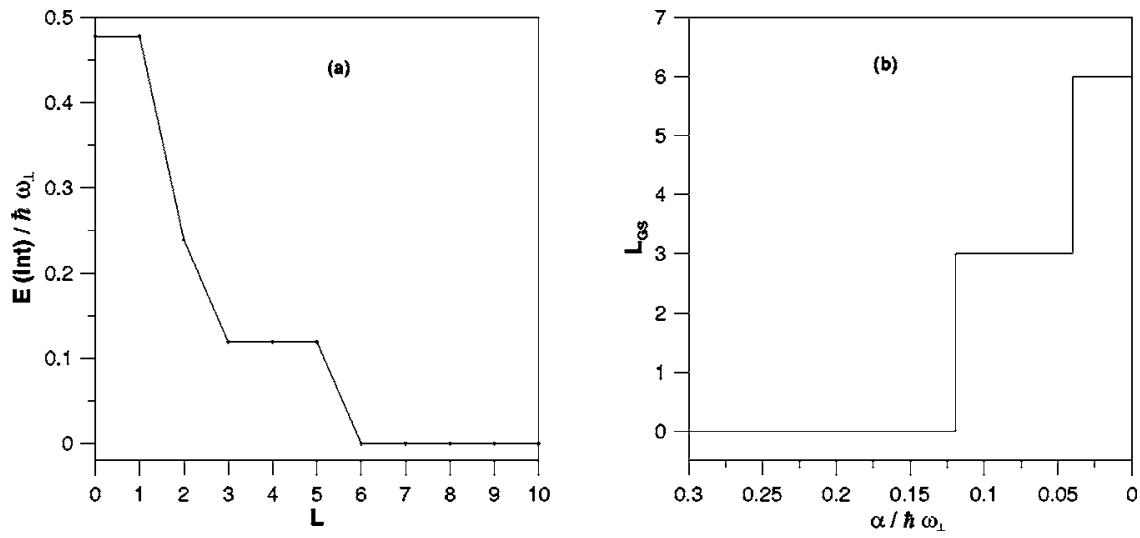

FIG. 5. For $N=3$, (a) lowest value of the interaction contribution to the energy as a function of total angular momentum (Yrast line). (b) Angular momentum of the GS over $\alpha$ [see Eq. (3)]. The critical values for $\alpha / \hbar \omega_{\perp}$ at the steps are 0.1194 and 0.0398 .

otherwise specified. We consider $\lambda$ and $\hbar \omega_{\perp}$ as units of length and energy unless otherwise stated.

For $N \gg 1$, the first vortex is nucleated at $\Omega_{c 1}=\omega_{\perp}$ $-g N /(8 \pi)$ when the transition from $L=0$ to $L=N$ takes place at the first step of $L_{g s}(\Omega)$. The $L=N$ state whith a vortex at the center is the GS from $\Omega_{c 1}$ to $\Omega_{c 2}$ (see Fig. 1). This state is characterized by a high occupancy of the $m=1 s p$ state and circular symmetry possessing no space correlations. In contrast, for small values of $N$ a vortex is not clearly manifested unless a considerably large number of atoms is considered, as it is shown in Fig. 3, where the pc of the $L=N$ state is displayed for $N=3,10$, and 20 . In all the cases, the density has a minimum at the origin and $r_{0}$ is set to its maximum. The slow tendency to recover the behavior of large condensed systems is explained in Fig. 4, where the occupation of the $m=1 s p$ state over $N$ is shown. Namely, for few atoms, the angular momentum of the state is not fully due to vortices. For a large number of atoms, Nakajima and Ueda have analyzed the formation of the first vortex, from its initial nucleation at the cloud boundary towards its final stabilization at the center [51]; our results share some similarity with this situation: for small $N$, the vortex is not yet fully inside the trap, and as $N$ increases, it approaches the trap center from the boundary of the cloud.

In Figs. 5-8, we show the main results for $N=3$ including the ground state evolution as $\Omega$ increases. Figure 5(a) shows the lowest eigenenergies for each $L$, the so-called Yrast line. The initial points of the plateaus, at $L=0,3$, and 6 are the unique possible GSs (besides degeneracies at the steps). A general result is that the plateau previous to the Lauhglin state (from $L=3$ to $L=5$ in this case) always has $N$ points. Figure 5(b) is similar to Fig. 1 for $N=5$. The densities from $L=0$ to 9 are shown in Fig. 6. The parameter $r_{0}$ used in the pc calculation was set to the maximum of the density unless it is located at the center. Then, $r_{0}=1$ is used if not stated otherwise. Figure 7 displays the pc function for $L=0,3,4,5$, 6 , and 9. The system evolves from a completely "condensed" system at $L=0$ to the Laughlin state at $L=6$ where a clear triangular structure appears. The loss of condensation is related to the increase of space correlations. To complete the analysis, we show in Fig. 8 the evolution of $s p$ occupations, and demonstrate that "macroscopic" occupation of a specific $s p$ wave function vanishes as $L$ increases.

In order to see how the previous general tendency evolves as $N$ increases, we analyzed the $N=5$ case. In Fig. 9 we show the densities of the GSs from $L=0$ to the Laughlin state, and in Fig. 10 we display their pc. The same tendency towards space ordering at the Laughlin state is clear. In addition, it can be inferred from the $L=20$ case that correlations are stronger for nearest neighbors as a manifestation of partial long range order in finite systems. In Fig. 11 the occupations of $s p$ states are shown. It is remarkable that some indications of the Laughlin state typical for large systems, i.e., a flat density at the central part and a hump at the edge, are already manifested in such small systems, as it can be seen in the last graphs of Figs. 11 and 9, respectively. Moreover, the density at the origin is very close to $1 /(2 \pi)=0.16$, as necessary for a homogeneous system at filling factor $1 / 2$.

In Figs. 12 and 13, we concentrate on the Laughlin state for $N=3,4,5,6,7$, and 8 atoms. The right-hand side picture

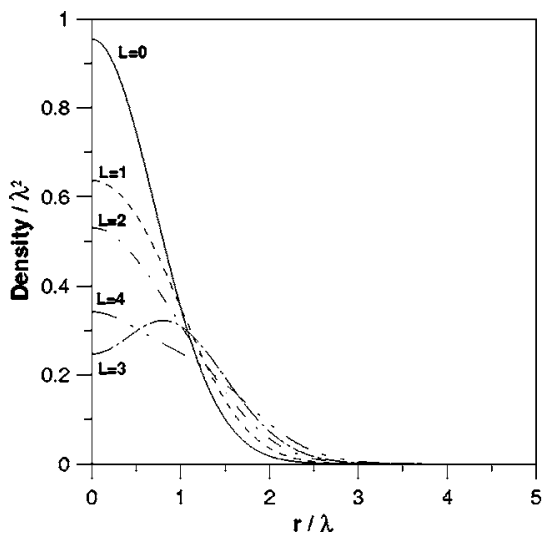

FIG. 6. $N=3$, density of the $L$-states. 

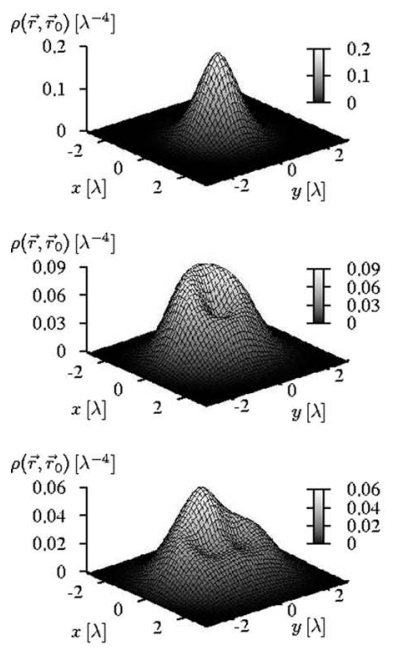
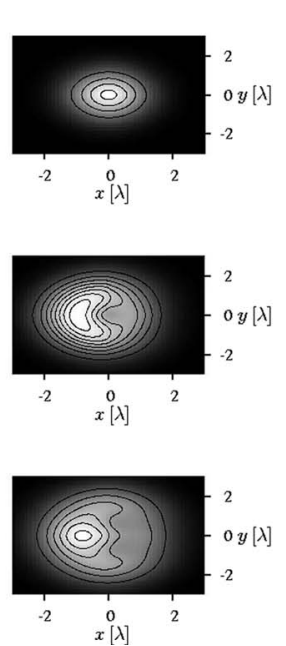
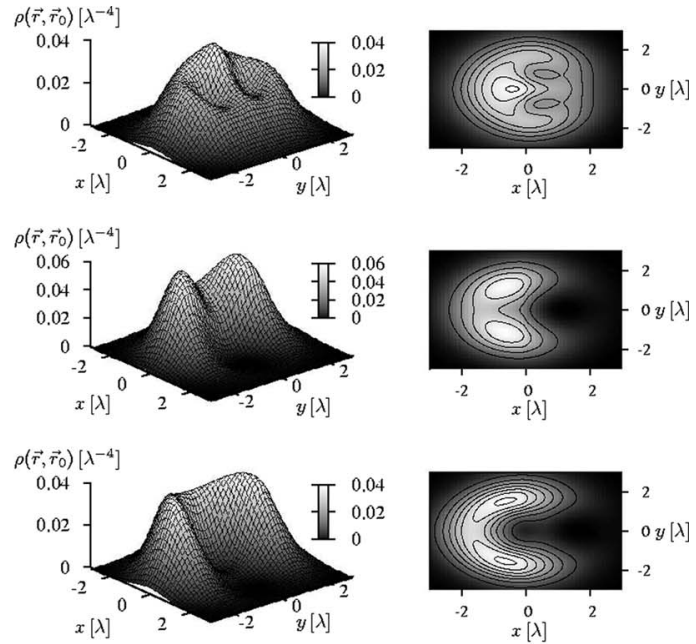

FIG. 7. The same as Fig. 3 for $N=3$, and $L=0,3$, and 4 on the left-hand side and from top to bottom and for $L=5,6$, and 9 on the right-hand side and from top to bottom. $r_{0}=1.0,0.8,1.0,1.0,1.3$, and 1.7 in units of $\lambda$, respectively.

for $N=3,4$, and 5 contains the pc function and the left-hand side displays the density of the superposition made from $L$ and $L+N$. This superposition of quasidegenerated states becomes a possible realization of a GS for a certain value of $\Omega$, as commented previously. The value of the parameter $r_{0}$ in the pc functions was obtained in a different way as the one used previously. Taking advantage of the fact that the Laughlin wave function [Eq. (6)] is the exact solution, and knowing that its $\mathrm{pc}$ shows a ring shape structure of an unknown radious $r_{0}$, we can maximize the probalibity distribution given by

$$
\left|\Psi_{\text {Laughlin }}\left(\vec{r}_{1}, \vec{r}_{2}, \ldots, \vec{r}_{N}\right)\right|^{2}=e^{-T},
$$

where

$$
T=\sum_{i} \frac{r_{i}^{2}}{2 \lambda^{2}}-2 q \sum_{i<j} \ln \left|z_{i}-z_{j}\right|
$$

(with $q=2$ for the bosonic Laughlin state), or equivalently, minimize $T$ with respect to $r_{0}$. Minimization yields $r_{0}=\sqrt{N-1}$ (or $r_{0}=\sqrt{N}$ if one atom is at the origin as for $N=6,7$, and 8) which is always smaller than the size of the
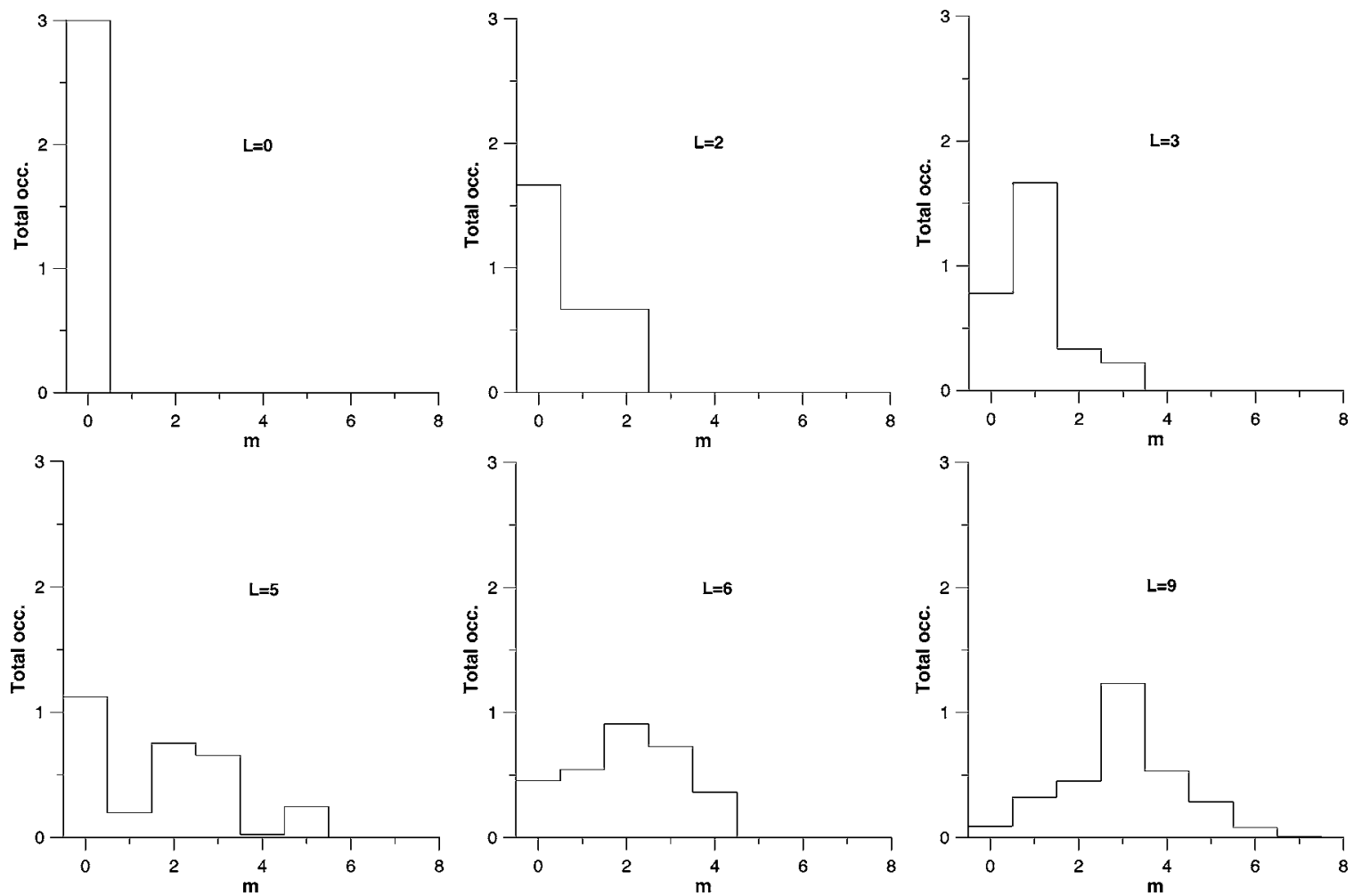

FIG. 8. For $N=3$, total occupations of the single particle states of angular momentum $m$ for several $L$-states. 

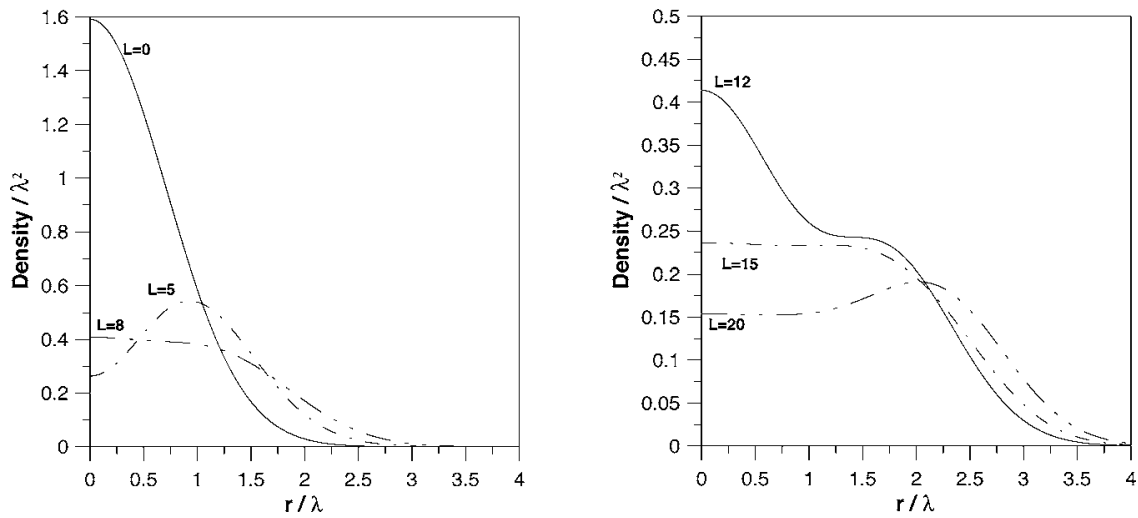

FIG. 9. $N=5$ density of the $L$-states (GS). system given by $R=\sqrt{4 N-2}$. To see the evolution of these hidden ordered structures as $N$ increases, the "degree of correlation" $C$ as a function of $N$ is displayed in Fig. 14; $C$ is defined as the height of the maximum peak in the pc function. It decreases for increasing $N$ as it is expected in order to recover the quantum liquid character of the Laughlin state for large systems.

Suggested by our results, a possible explanation for the realization of Wigner molecules in the Laughlin states for few bosons is the following. The first observation is that the nature of the GS does not depend on the kinetic part as the diagonalization is fully determined by the interaction, in other words, the structure does not result from the competition between different kinds of energy. In addition, as the repulsive interaction energy is zero in the Laughlin state, it seems that the reason why the atoms choose symmetric and well-separated positions is due to two conditions, first, the system must have a large angular momentum given by $L$ $=N(N-1)$ (which means large distances from the origin) and second, each atom is surrounded by a quasihole (which leads to effective mutual repulsion). This last statement is supported by the following observation. The contour plots of $N=3$ for $L=4$ and $L=5$ in Fig. 7 suggest that in those precursory states (the Laughlin state has $L=6$ ), quasiholes not attached to atoms are created without cost of internal energy, the contribution to the angular momentum of each one would evolve as $1 / 3$ (in $L=4$ ), 2/3 (in $L=5$ ), and $3 / 3$ until the Laughlin structure becomes possible with one quasihole attached to each atom, lowering the interaction energy. A final observation relates to the evolution of this behavior as $N$ increases. Due to the fact that the dependence of $L$ on $N$ is quadratic, the increase of $L$ with $N$ is more efficient for large $N$, and atoms do not have to be widely separated. Thus the symmetric distribution tends to disappear. Recently, it has been proposed that the phenomenology of strongly correlated bosonic and fermionic rotating systems converges to the case of classical particles, and finally crystallizes at high rotational frequencies [63]. Our results exhibit some traces of such crystallization, but it should be pointed out that this "crystallization" ceases to be manifested when the bulk structure starts to dominate the system. This happens for bigger particle numbers, where the GS at Laughlin angular momentum will start to behave more and more like a true quantum liquid as pointed out above.

For $N=5$ and 6, Fig. 15 shows patterns of two incipient vortices obtained from full diagonalization of $H+V_{p}$ at $\hbar\left(\omega_{\perp}-\Omega\right)=0.0458$ and $\hbar\left(\omega_{\perp}-\Omega\right)=0.05904$ in units of $\hbar \omega_{\perp}$, respectively (at the third step in both cases). The possibility to obtain these patterns for the given anisotropy strongly depends on the possibility to obtain truly degenerate states
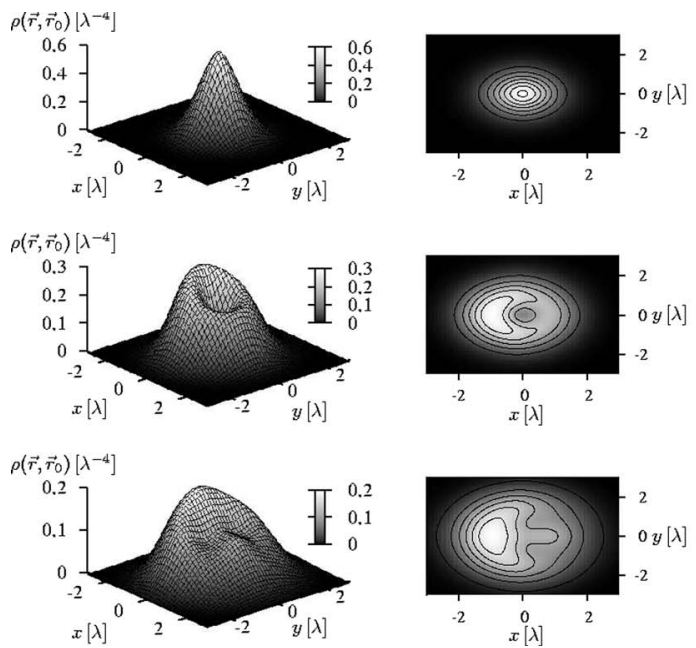
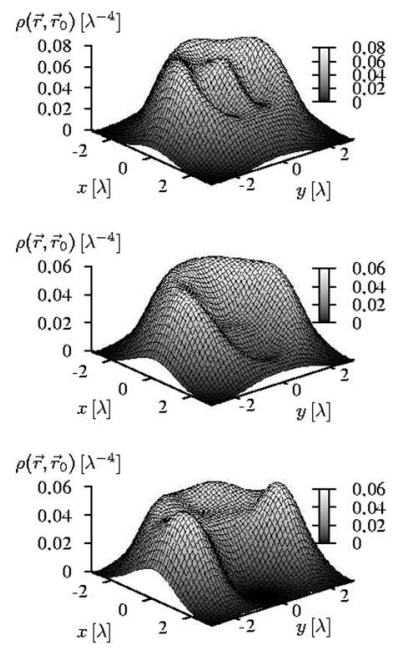
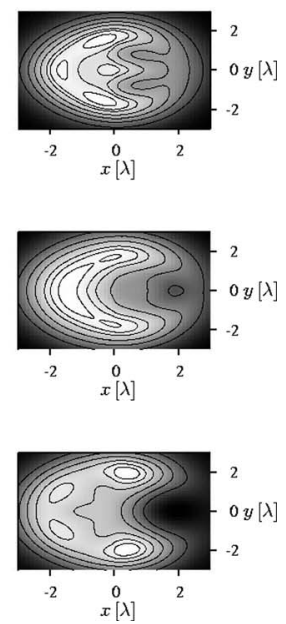

FIG. 10. The same as Fig. 7 for $N=5$ and $L=0,5$, and 8 on the left-hand side and $L=12,15$, and 20 on the right-hand side. $r_{0}=1.0,0.9$, $1.0,1.0,1.0$, and 2.0 in units of $\lambda$, respectively. 

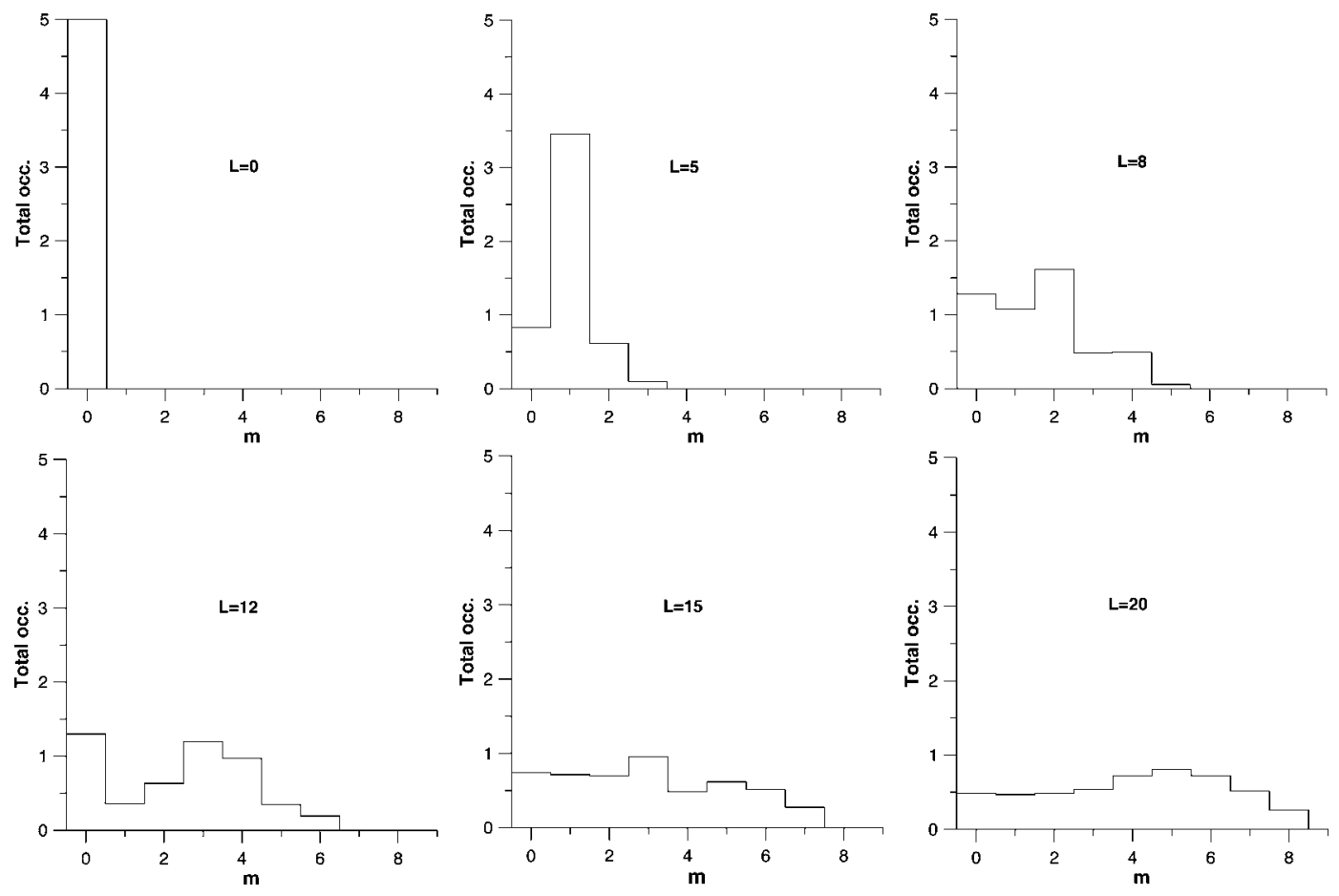

FIG. 11. $N=5$ occupations of the $L$-states (GS).

with angular momenta $L$ and $L \pm 2$ at one of the steps $L_{g s}(\Omega)$. For $N=7$ we were not able to find such steps. For $N=5$ the result of the diagonalization at the third step, where $L=8,10$, and 12 are involved, is that the weights of $L=8$ and 10 within the expansion of the GS are much larger that the
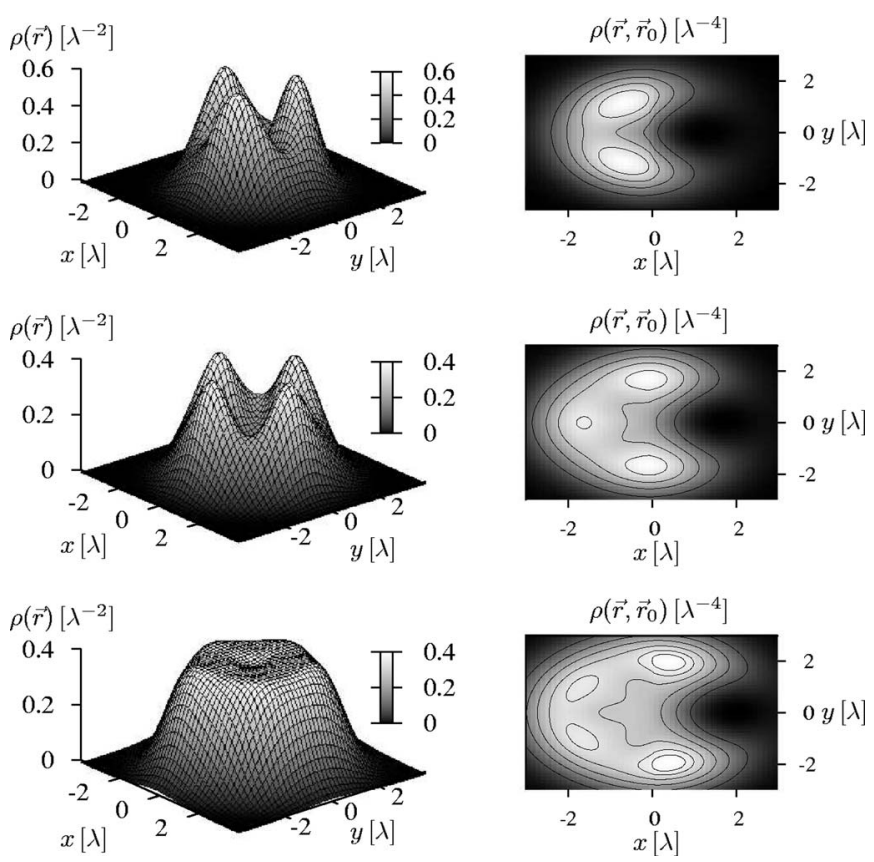

FIG. 12. For $N=3,4$, and 5 the left-hand side 3D plots show the density of the mixtures (with equal coefficients) of angular momenta $6+9,12+16$, and $20+25$, respectively. The right-hand side shows the pair correlation function (contour-plots) for $L=6,12$, and 20, respectively (the Laughlin states), with $r_{0}=\sqrt{N-1} / \lambda$. weight of $L=12$. This leads in effect to a state with expected vortex angular momentum lower than 10 , in agreement with the results demonstrated in Ref. [52] related to the fact that the contribution of a vortex to the total angular momentum depends on its distance from the origin, it runs from $N$ at the center to zero at the trap boundary.
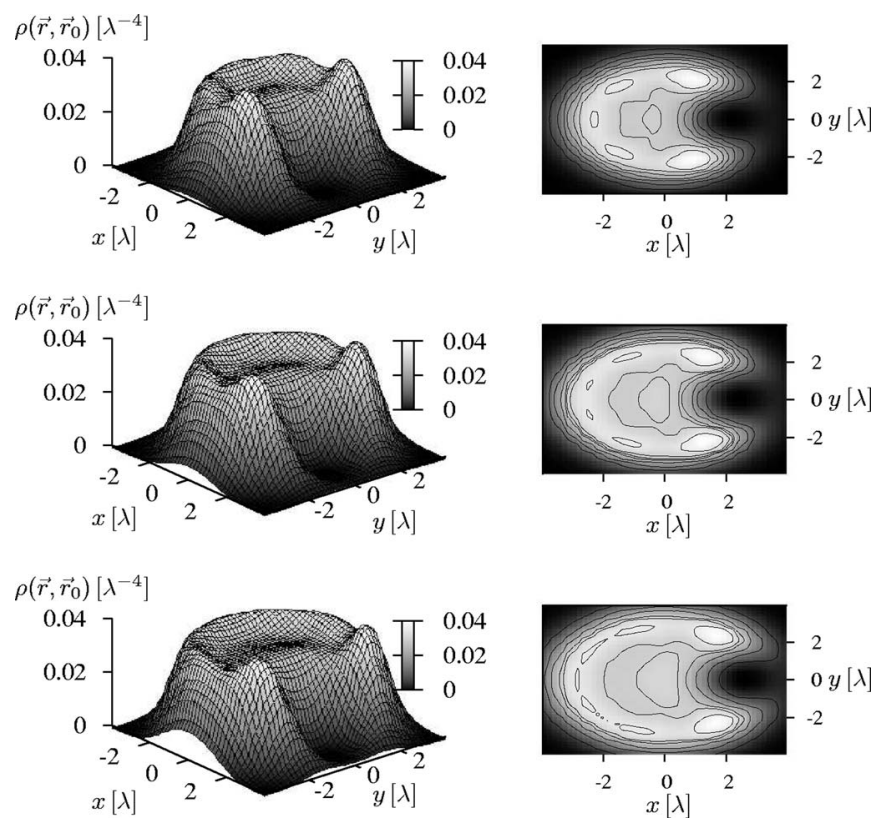

FIG. 13. The same as Fig. 3 for $N=6,7$, and 8 and for $L=30$, 42 , and 56, respectively (the Laughlin states). We consider $r_{0}=\sqrt{N} / \lambda$. 


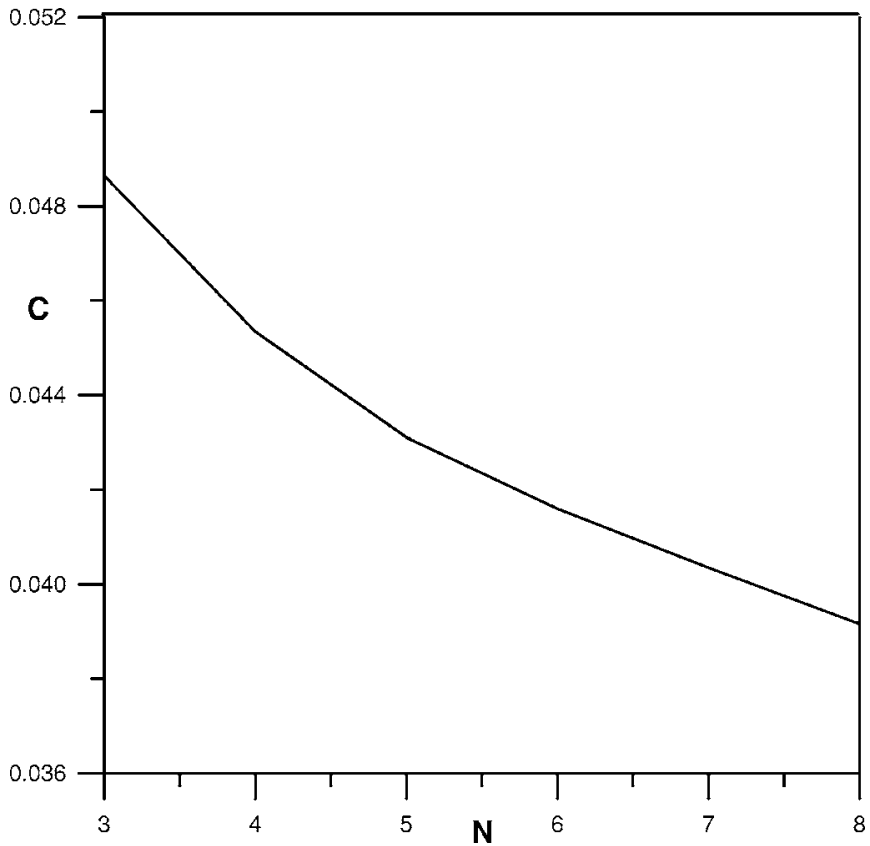

FIG. 14. Correlation degree (see text) of the Laughlin state as a function of $N$.

In accordance with the results commented in the previous paragraph, in order to generate incipient vortices in the density distribution, the value of $\Omega$ must be carefully tuned around $\Omega_{c n}$. We were not able to generate vortices for values of $\Omega$ comprised within the interval ranging from $\Omega_{c n}$ to $\Omega_{c n+1}$. This would have important experimental consequences in the dependence of the vortex contribution to the angular momentum as a function of $\Omega$. While this function is monotonously increasing for large systems, we expect it to become a function with peaks around $\Omega_{c n}$ and wide minima in between for small systems. As $N$ increases, the peaks would broaden due to the appearance of microplateaus (shown in Fig. 2) which in turn lead to finite ranges of $\Omega$-values where quasidegenerate states coexist. In this
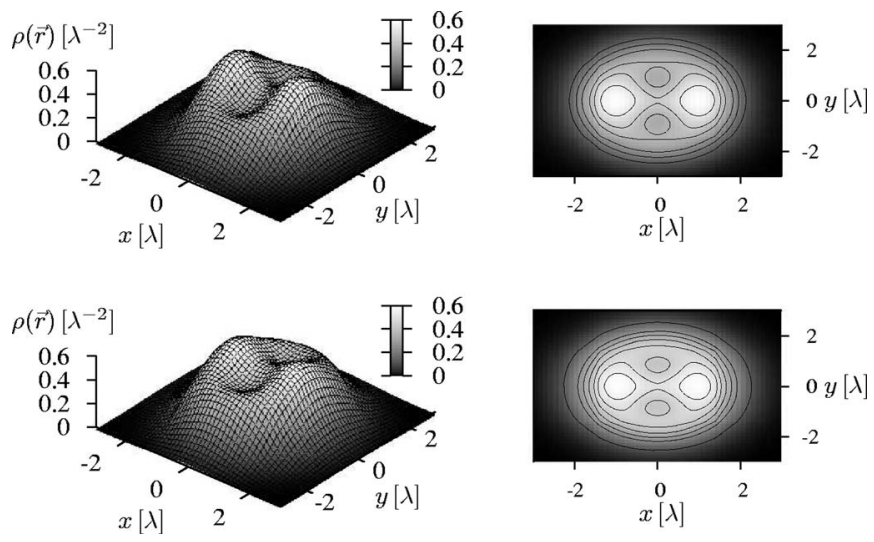

FIG. 15. For $N=5$ and 6 density (3D plot and contour plot) of two vortex structures. For $N=5, L=8+10+12$ and for $N=6$, $L=10+12+14$ (see text). manner the experimental result for large systems would be recovered. It is to be noted that in all our calculations we considered $g \lambda^{-2}=1$ in units of $\hbar \omega_{\perp}$ which is large compared with typical experimental values used for large systems $\left(g \lambda^{-2} / \hbar \omega_{\perp}=0.02\right.$ would be obtained by using data from Refs. $[53,54]$ for ${ }^{87} \mathrm{Rb}$ ). However, the effect of a reduced $g$ is solely a change of scale with respect to the $x$ axis in Fig. 2 which does not affect any previously obtained conclusions.

\section{SUMMARY}

The main result of this paper is the identification of important differences between large and small systems of rapidly rotating cold bosonic atoms. These differences can be understood by looking at the expected values of the density and the pc functions, on which we have concentrated our analysis. The characterization of small samples in rotating traps has recently attracted increasing interest due to the possibility to deal with a small number of atoms per well in optical lattices [46].

Within the regime of low rotational frequency, we obtain that a relatively large number of atoms is necessary to nucleate the first vortex carrying $N$ units of angular momentum. The evolution towards the condensed state with $L=N$ is shown by the increase of the occupation of the $s p m=1$ state as $N$ increases. On the other hand, in the regime of strong rotation, space correlations increase significantly, and in the Laughlin state a hidden ordered structure modulates the pc pattern. For small systems, the atoms sit around a ring of radius $r_{0}=\sqrt{N-1}\left(N=3,4\right.$, and 5) or $r_{0}=\sqrt{N}(N=6,7$, and 8$)$. The degree of correlation defined as the height of the peaks in the ordered pattern decreases with $N$, evolving towards a noncorrelated structure of a quantum liquid. We have argued about the observability of the ordered IP in similar experiments as those reported, for instance, by Fölling et al. [22].

Numerous references have analyzed the Wigner structures of few electrons [55-59]; Yannouleas and Landman (cf. [60]) and $\mathrm{Li}$ et al. (cf. [61]) have extensively investigated these ordered structures exhibited in the pc function for several filling factors including $1 / 3$ and obtain that for electrons, the crystallization of the ground state is much stronger than that exhibited by the Laughlin wave function. It is important to remark that it is well-established from exact diagonalization studies in a torus geometry and from the analysis of the Laughlin wave function, that states of filling factor $1 / 3$ for electrons and $1 / 2$ for bosons are fermionic quantum liquids in the thermodynamic limit (cf. [62]). Whenever the Laughlin function is a good approximation, its implied properties are independent from the interaction. However, for small confined systems the previous results do not apply, and the analysis of some of their properties relies on the competition between the kinetic and interaction energies, aside from their statistics.

Finally, precursors of two-vortex arrays are obtained as the ground states of an asymmetric Hamiltonian that models the experimental setup used to increase the angular 
momentum of a trapped Bose condensate by a stirring laser. We conclude that the possibility to nucleate vortex patters in the density is restricted for small $N$ to the specific values $\Omega_{c n}$ at the steps where several degenerate or quasidegenerate states of different angular momentum $L$ coexist. This produces peaks in the vortex angular momentumdependence on $\Omega$. We predict these peaks to broaden as $N$ increases, due to the appearance of "microplateaus" which in turn lead to finite ranges of $\Omega$ values where quasidegenerate states coexist.

\section{ACKNOWLEDGMENTS}

We thank J. Dalibard, L. Pitaevskii, G.V. Shlyapnikov, and S. Stringari for fruitful discussions. We acknowledge support from the Deutsche Forschungsgemeinschaft (SFB 407, SPP 1116, GK 282, 436 POL), the EU Programme QUPRODIS, ESF PESC QUDEDIS, EU IP Programme "SCALA," and the MEC (Spanish Goverment) in terms of the contracts FIS2005-04627 and FIS2004-05639 and 2005SGR00343 from Generalitat de Catalunya.
[1] M. H. Anderson, J. R. Ensher, M. R. Matthews, C. E. Wierman, and E. A. Cornell, Science 169, 198 (1995).

[2] K. B. Davis, M.-O. Mewes, M. R. Andrews, N. J. van Druten, D. S. Durfee, D. M. Kurn, and W. Ketterle, Phys. Rev. Lett. 75, 3969 (1995).

[3] C. C. Bradley, C. A. Sackett, and R. G. Hulet, Phys. Rev. Lett. 78, 985 (1997); C. C. Bradley, C. A. Sackett, J. J. Tollett, and R. G. Hulet, ibid. 75, 1687 (1995).

[4] L. Pitaevskii and S. Stringari, Bose-Einstein Condensation (Oxford Science Publishers, Oxford, 2003).

[5] M. R. Andrews, C. G. Townsend, H. J. Miesner, D. S. Durfee, D. M. Kurn, and W. Ketterle, Science 275, 637 (1997).

[6] Juha Javanainen and Sung Mi Yoo, Phys. Rev. Lett. 76, 161 (1996); J. Javanainen and M. Wilkens, ibid. 78, 4675 (1997).

[7] Y. Castin and J. Dalibard, Phys. Rev. A 55, 4330 (1997); for a review see P. Villain, M. Lewenstein, R. Dum, Y. Castin, L. You, A. Imamoglu, and T. A. B. Kennedy, J. Mod. Opt. 44, 1775 (1997).

[8] J. Dziarmaga, Z. P. Karkuszewski, and K. Sacha, Phys. Rev. A 66, 043615 (2002).

[9] K. W. Madison, F. Chevy, W. Wohlleben, and J. Dalibard, Phys. Rev. Lett. 84, 806 (2000).

[10] J. R. Abo-Shaeer, C. Raman, J. M. Vogels, and W. Ketterle, Science 292, 476 (2001).

[11] A. Abrikosov, Zh. Eksp. Teor. Fiz. 32, 1442 (1957) [Sov. Phys. JETP 5, 1174 (1957)].

[12] In experiments of the ENS group, for instance, a laser beam forms a Gaussian optical potential. It rapidly oscillates through the trap center along the direction that slowly rotates around the trap center. Obviously, such a process couples states with various angular momenta.

[13] See for instance, G. Baym, Acta Phys. Pol. B 29, 1839 (1998), and references therein.

[14] R. H. Brown and R. Q. Twiss, Nature (London) 178, 1046 (1956).

[15] M. Yasuda and F. Shimizu, Phys. Rev. Lett. 77, 3090 (1996);A. Öttl, S. Ritter, M. Köhl, and T. Esslinger, Phys. Rev. Lett. 95, 090404 (2005).

[16] M. Schellekens, R. Hoppeler, A. Perrin, J. V. Gomes, D. Boiron, A. Aspect, and C. I. Westbrook, Science 310, 648 (2005).

[17] D. Hellweg, L. Cacciapuoti, M. Kottke, T. Schulte, K. Sengstock, W. Ertmer, and J. J. Arlt, Phys. Rev. Lett. 91, 010406 (2003); L. Cacciapuoti, D. Hellweg, M. Kottke, T. Schulte, W. Ertmer, J. J. Arlt, K. Sengstock, and L. Santos, Phys. Rev. A 68, 053612 (2003).
[18] E. Altman, E. Demler, and M. D. Lukin, Phys. Rev. A 70, 013603 (2004).

[19] N. Read and N. R. Cooper, Phys. Rev. A 68, 035601 (2003).

[20] R. Bach and K. Rzążewski, Phys. Rev. Lett. 92, 200401 (2004); R. Bach and K. Rzążewski, Phys. Rev. A 70, 063622 (2004).

[21] Z. Hadzibabic, S. Stock, B. Battelier, V. Bretin, and J. Dalibard, Phys. Rev. Lett. 93, 180403 (2004).

[22] S. Fölling, F. Gerbier, A. Widera, O. Mandel, T. Gericke, and I. Bloch, Nature (London) 434, 481 (2005).

[23] M. Greiner, C. A. Regal, J. T. Stewart, and D. S. Jin, Phys. Rev. Lett. 94, 110401 (2005).

[24] D. M. Stamper-Kurn, A. P. Chikkatur, A. Görlitz, S. Inouye, S. Gupta, D. E. Pritchard, and W. Ketterle, Phys. Rev. Lett. 83, 2876 (1999); J. Stenger, S. Inouye, A. P. Chikkatur, D. M. Stamper-Kurn, D. E. Pritchard, and W. Ketterle, ibid. 82, 4569 (1999).

[25] N. K. Wilkin, J. M. F. Gunn, and R. A. Smith, Phys. Rev. Lett. 80, 2265 (1998); N. K. Wilkin and J. M. F. Gunn, Phys. Rev. Lett. 84, 6 (2000).

[26] B. Paredes, P. Fedichev, J. I. Cirac, and P. Zoller, Phys. Rev. Lett. 87, 010402 (2001)

[27] R. B. Laughlin, Phys. Rev. Lett. 50, 1395 (1983).

[28] R. E. Prange and S. M. Girvin, The Quantum Hall Effect (Springer-Verlag, Berlin, 1990).

[29] N. R. Cooper, N. K. Wilkin, and J. M. F. Gunn, Phys. Rev. Lett. 87, 120405 (2001); J. Sinova, C. B. Hanna, and A. H. MacDonald, ibid. 89, 030403 (2002); N. Regnault and T. Jolicoeur, Phys. Rev. B 70, 241307(R) (2004).

[30] N. Read and E. Rezayi, Phys. Rev. B 59, 8084 (1999).

[31] G. Moore and N. Read, Nucl. Phys. B 360, 362 (1991); M. A. Cazalilla, N. Barberan, and N. R. Cooper, Phys. Rev. B 71, 121303 (2005)

[32] A. G. Morris and D. L. Feder, e-print cond-mat/0602037.

[33] I. Coddington, P. Engels, V. Schweikhard, and E. A. Cornell, Phys. Rev. Lett. 91, 100402 (2004).

[34] V. Schweikhard, I. Coddington, P. Engels, S. Tung, and E. A. Cornell, Phys. Rev. Lett. 93, 210403 (2004).

[35] V. Schweikhard, I. Coddington, P. Engels, V. P. Mogendorff, and E. A. Cornell, Phys. Rev. Lett. 92, 040404 (2002); T. P. Simula, P. Engels, I. Coddington, V. Schweikhard, E. A. Cornell, and R. J. Ballagh, ibid. 94, 080404 (2005); S. Stock, B. Battelier, V. Bretin, Z. Hadzibabic, and J. Dalibard, Laser Phys. Lett. 2, 275 (2005).

[36] M. Baranov, L. Dobrek, K. Góral, L. Santos, and M. Lewenstein, Phys. Scr. 102, 74 (2002). 
[37] N. R. Cooper, E. H. Rezayi, and S. H. Simon, Phys. Rev. Lett. 95, 200402 (2005).

[38] M. A. Baranov, K. Osterloh, and M. Lewenstein, Phys. Rev. Lett. 94, 070404 (2005).

[39] J. Stuhler, A. Griesmaier, T. Koch, M. Fattori, T. Pfau, S. Giovanazzi, P. Pedri, and L. Santos, Phys. Rev. Lett. 95, 150406 (2005).

[40] See, e.g., special issue on ultracold polar molecules [Eur. Phys. J. D 31, 149 (2004)].

[41] D. Jaksch and P. Zoller, New J. Phys. 5, 56 (2003); E. J. Mueller, Phys. Rev. A 70, 041603(R) (2004); A. S. Sorensen, E. Demler, and M. D. Lukin, Phys. Rev. Lett. 94, 086803 (2005); K. Osterloh, M. Baig, L. Santos, P. Zoller, and M. Lewenstein, ibid. 95, 010403 (2005).

[42] J. Ruseckas, G. Juzeliunas, P. Öhberg, and M. Fleischhauer, Phys. Rev. Lett. 95, 010404 (2005).

[43] I. Bloch (private communication).

[44] G. Birkl (private communication); see also G. Birkl, F. B. J. Buchkremer, R. Dumke, and W. Ertmer, Opt. Commun. 191, 1 (2001).

[45] D. D. Yavuz, P. B. Kulatunga, E. Urban, T. A. Johnson, N. Proite, T. Henage, T. G. Walker, and M. Saffman, Phys. Rev. Lett. 96, 063001 (2006).

[46] M. Popp, B. Paredes, and J. I. Cirac, Phys. Rev. A 70, 053612 (2004).

[47] F. Chevy, K. W. Madison, and J. Dalibard, Phys. Rev. Lett. 85, 2223 (2000).

[48] M. A. Cazalilla, Phys. Rev. A 67, 063613 (2003).

[49] L. Jacak, P. Hawrylak, and A. Wojs, Quantum Dots (SpringerVerlag, Berlin, 1997).

[50] Such a model describes the experiments of Ref. [9] quite well, and has several different advantages: (i) it has a simple analytical form and allows for simple calculations; and (ii) it also describes other kinds of "stirring," for instance, the one in which one uses an elliptical, rotating mask to generate the optical potential of Eq. (17).

[51] T. Nakajima and M. Ueda, Phys. Rev. Lett. 91, 140401 (2003).

[52] F. Zambelli and S. Stringari, Phys. Rev. Lett. 81, 1754 (1998).

[53] I. Danaila, Phys. Rev. A 72, 013605 (2005).

[54] W. Bao, D. Jaksch, and P. A. Markowich, J. Comput. Phys. 187, 318 (2003).

[55] K. Jauregui, W. Häusler, and B. Kramer, Europhys. Lett. 24, 581 (1993).

[56] K. Yang, F. D. M. Haldane, and E. H. Rezayi, Phys. Rev. B 64, 081301(R) (2001).

[57] N. Shibata and D. Yoshioka, Physica E (Amsterdam) 22, 111 (2004)

[58] E. Rasanen, H. Saarikoski, M. J. Puska, and R. M. Nieminen, Phys. Rev. B 67, 035326 (2003).

[59] B. Szafran, F. M. Peeters, S. Bednarek, T. Chwiej, and J. Adamowski, Phys. Rev. B 70, 035401 (2004); B. Szafran, T. Chwiej, F. M. Peeters, S. Bednarek, and J. Adamowski, Phys. Rev. B 71, 235305 (2005).

[60] C. Yannouleas and U. Landman, Phys. Rev. B 70, 235319 (2004).

[61] Y. Li, C. Yannouleas, and U. Landman, Phys. Rev. B 73, 075301 (2006).

[62] D. Yoshioka, The Quantum Hall Effect (Springer-Verlag, Berlin, 2002).

[63] S. M. Reimann, M. Koskinen, Y. Yu, and M. Manninen, New J. Phys. 8, 59 (2006) 